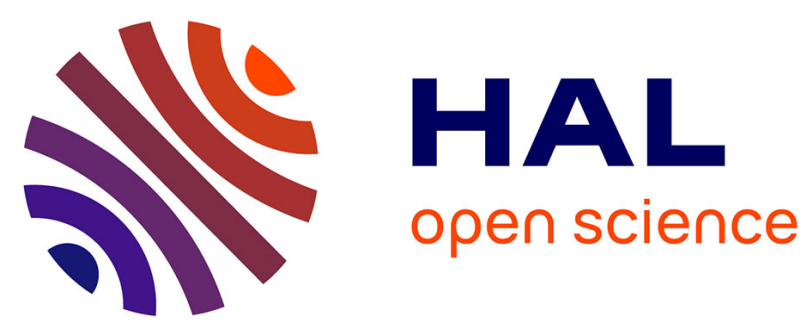

\title{
Vibration Analysis of an Axial Turbine Blisk with Optimized Intentional Mistuning Pattern
}

Bernd Beirow, Felix Figaschewsky, Arnold Kühhorn, Alfons Bornhorn

\section{To cite this version:}

Bernd Beirow, Felix Figaschewsky, Arnold Kühhorn, Alfons Bornhorn. Vibration Analysis of an Axial Turbine Blisk with Optimized Intentional Mistuning Pattern. 17th International Symposium on Transport Phenomena and Dynamics of Rotating Machinery (ISROMAC2017), Dec 2017, Maui, United States. hal-02397955

\section{HAL Id: hal-02397955 https://hal.science/hal-02397955}

Submitted on 6 Dec 2019

HAL is a multi-disciplinary open access archive for the deposit and dissemination of scientific research documents, whether they are published or not. The documents may come from teaching and research institutions in France or abroad, or from public or private research centers.
L'archive ouverte pluridisciplinaire HAL, est destinée au dépôt et à la diffusion de documents scientifiques de niveau recherche, publiés ou non, émanant des établissements d'enseignement et de recherche français ou étrangers, des laboratoires publics ou privés. 


\title{
Vibration Analysis of an Axial Turbine Blisk with Optimized Intentional Mistuning Pattern
}

\author{
Bernd Beirow $^{1 *}$, Felix Figaschewsky ${ }^{1}$, Arnold Kühhorn ${ }^{1}$, Alfons Bornhorn ${ }^{2}$

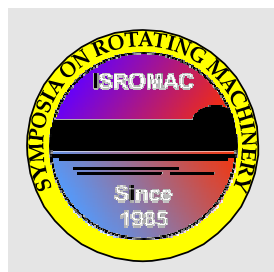 \\ ISROMAC 2017 \\ International \\ Symposium on \\ Transport \\ Phenomena and \\ Dynamics of

\section{Abstract} \\ Aiming to limit the forced response of an axial turbine blisk for ship Diesel engine applications \\ efforts have been made to increase the aerodynamic damping contribution for the most critical modes. \\ In this regard the potential of intentional mistuning is investigated since it offers the opportunity to \\ ensure a safe operation without a severe loss of aerodynamic performance. Genetic algorithms have \\ been chosen to derive an optimized mistuning pattern. In order to keep the manufacturing effort within \\ a limit only two possible blade geometries are allowed which means that an integer optimization \\ problem has been formulated. For the purpose of demonstrating the benefit of the intentional mistuning \\ pattern found, two blisk prototypes have been manufactured: One with and another one without \\ employing intentional mistuning for purposes of comparison. Furthermore, this offers the opportunity for \\ an experimental determination of mistuning being really manufactured and other modal properties as \\ well. The experimental data basis is employed to update structural models which are well suited to \\ demonstrate the forced response reduction under operational conditions.
}

Rotating Machinery

Hawaii, Maui

December 16-21

2017

\author{
Keywords \\ Blisk — Intentional Mistuning — Low Engine Order Excitation \\ ${ }^{1}$ Chair of Structural Mechanics and Vehicle Vibration Technology, Brandenburg University of Technology, Cottbus, Germany \\ ${ }^{2}$ MAN Diesel \& Turbo SE, Augsburg, Germany \\ *Corresponding author: beirow@b-tu.de
}

\section{INTRODUCTION}

It is well known in the turbomachinery community that unavoidable variations of mechanical properties from blade to blade, which are denoted as mistuning, can cause frequency splitting and severe magnifications of the forced response compared to the regular design with identical blades. On that account researchers have been occupied with this problem for many years. In this regard the work of Whitehead ${ }^{[1]}$ deserves a special mentioning, since it allows for simply calculating a very conservative limit of the maximum response magnification, which only depends on the number of blades. Several years later, Martel and Corral [2] derived an enhanced and less conservative limit by taking into account the modal coupling within the blade mode family considered. Most recently Figaschewsky and Kühhorn [3] presented a further modification for calculating the worst expectable forced response by additionally considering the mistuning strength. Their approach is covering the before mentioned limits ${ }^{[1]}\left[{ }^{[2]}\right.$ as special cases. Petrov and Ewins ${ }^{[4]}$ as well as Chan ${ }^{[5]}$ did apply optimization algorithms to find mistuning patterns yielding the worst forced response. However, the maximum forced response given in the majority of publications (e.g. ${ }^{[6]}$ and ${ }^{[7]}$ ) is calculated by employing probabilistic methods [6] or really measured mistuning patterns ${ }^{[7]}$. Commonly, the maximum response magnification take values clearly below those computed in [1]- [5].

Regarding fundamental blade modes, in particular low engine order excitations (LEO) are well known to cause forced vibrations of blade integrated disks in turbocharger applications. Hence, LEOs have to be considered as a relevant source of fatigue. Recently, efforts have been spent to mitigate the forced response by means of employing intentional mistuning patterns. This has proved to be a promising way by both Petrov ${ }^{[8]}$ and Schoenenborn et al. [9] if first the dedicated travelling wave mode of the tuned counterpart is weakly damped compared to most of the other travelling wave modes and second that sufficiently high differences are existing between the maximum and the minimum of inter blade phase angle-dependent aerodynamic modal damping ratios. Similar results are achieved in ${ }^{[10]}$ for a high pressure compressor blisk, where a mistuning pattern has been derived by employing genetic algorithms which effects a forced response reduction down to a level of $52 \%$ compared to the perfect design with identical blades.

In this paper, genetic algorithms are applied as well to design an optimized intentional mistuning pattern for an axial turbine blisk with 41 blades namely to reduce the forced response of the fundamental flap mode clearly below that of the tuned counterpart without severely increasing the response of higher modes. Subset of nominal system mode (SNM) ${ }^{[11]}$ models are employed for that purpose in which the aeroelastic coupling is considered by means of aerodynamic influence coefficients (AIC) as suggested by Kahl [12]. Two prototypes have been manufactured to prove the suitability of the approach: A first one with the optimized intentional mistuning pattern (Fig. 1) and another one with theoretically identical blades. The necessary differences of blade alone frequencies from blade to blade in case of the intentionally 
mistuned blisk have been accomplished by intended geometry variations. Since additional random mistuning due to the process of manufacture is unavoidable, disparities in modal properties are expected between design intention and really manufactured blisk. That is why experimental analyses are carried out in order to identify the differences between intended designs and really manufactured blisks in terms of determining frequency based mistuning. Finally, updated structural models are used for numerical forced response analyses in order to prove the robustness of the intentional mistuning pattern with respect to additional random mistuning.

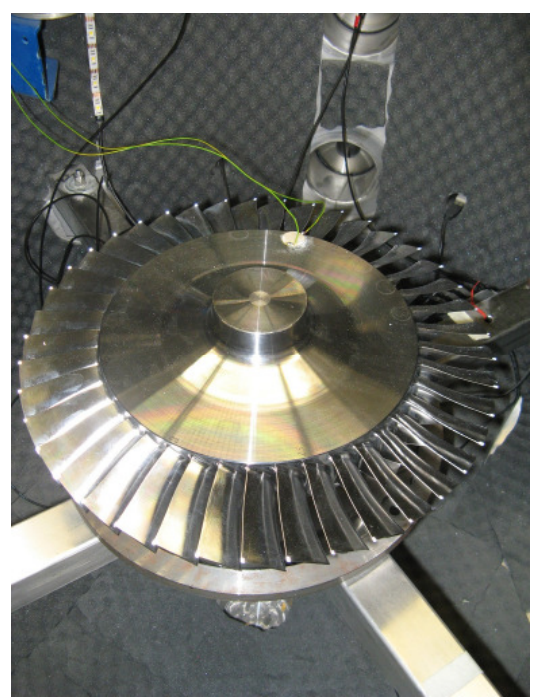

Figure 1. Blisk Prototype Inside Pressure Vessel

\section{BASIC VIBRATION ANALYSES}

Basic numerical analyses have been carried out by means of a finite element sector model (Fig. 2) with approximately 300.000 DOF in order to get both an overview about the basic natural vibration characteristics of the blisk with identical blades according to the original design and to prepare the input for SNM-models to be employed later on. Exemplary computational results (Fig. 3) are showing two typical blisk modes which are denoted as cyclic symmetry modes (CSM) 2 and 5 in compliance to the number of nodal diameter lines, which are separating sectors of identical size. If mistuning is present, the cyclic symmetry will get lost and the sectors will differ. As far as the original characteristic of the shape is still recognizable, such a mode is denoted as modified cyclic symmetry mode (MCSM). A further distinction of modes is affecting the blades, which are most sensitive with respect to possible forced responses due to aerodynamic excitation. It is common to group blade modes (Fig. 4) in blade mode families (BF). The natural frequencies of modes grouped in a BF are commonly differing in dependence on the modal coupling.

The Campbell diagram shown in Fig. 5 gives an impression about the most substantial resonance crossings of blade modes and engine orders (EO). The consideration of both rotational and temperature effects yield a slight decrease of the blade modes (solid black) with increasing engine speed. Preceding numerical analyses ${ }^{[13]}$ have shown that small perturbations of the nominal NGV-geometry are severely contributing to the forcing of the fundamental first blade mode (M1). Hence, the LEO-crossing occurring at $90 \%$ speed, namely EO6, is highlighted in Fig. 5 and considered in more detail in this paper.

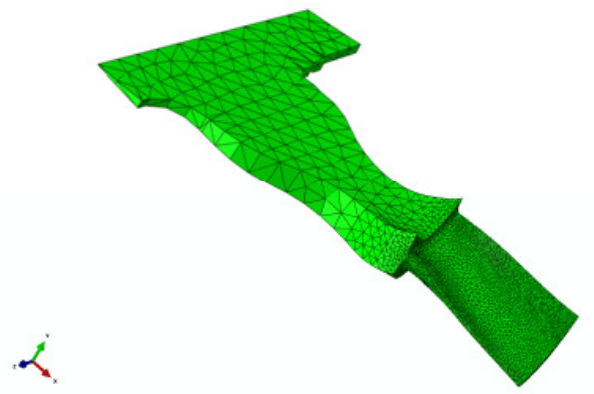

Figure 2. FE-Sector Model

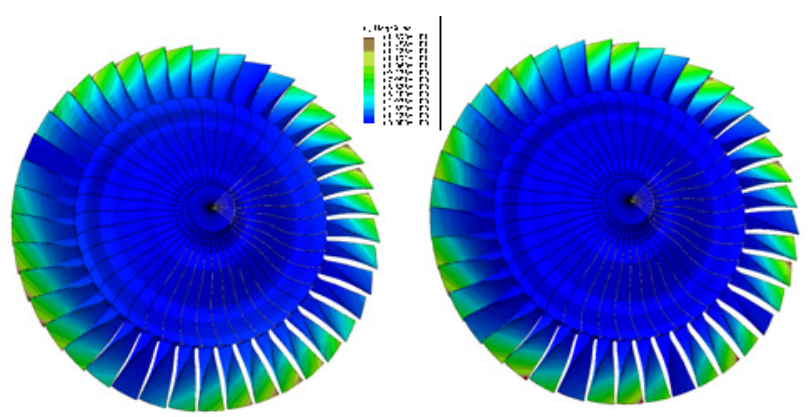

Figure 3. Magnitude Plots of CSM 2 and 5 (BF 1)

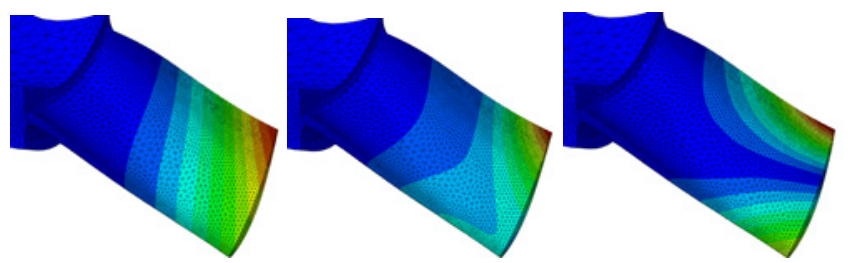

Figure 4. Blade Modes 1, 2 and 3

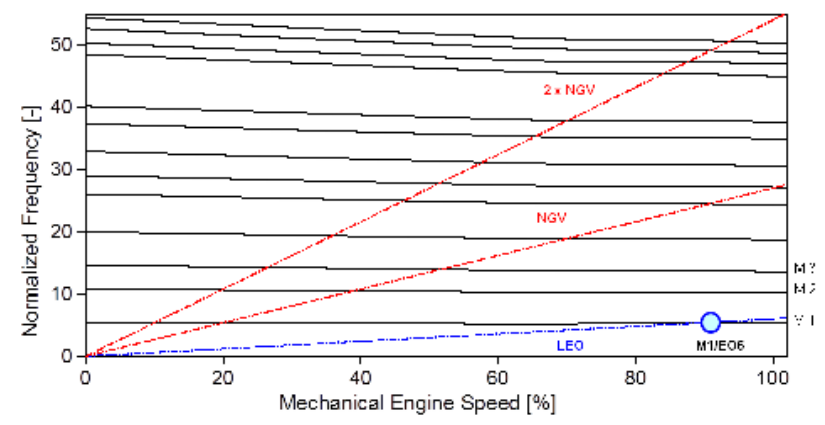

Figure 5. Campbell Plot 


\section{OPTIMIZATION OF INTENTIONAL MISTUNING PATTERN}

Aiming to find an optimized intentional mistuning pattern for a turbine blisk regarding the relevant EO 6 excitation of the first mode (M1) a minimization of the greatest possible forced response is formulated as objective function. In order to keep the manufacturing effort within a limit only two possible blade geometries are allowed which means that an integer optimization problem is formulated. Hence, the dimension of the design space takes a value of $2^{41}$ or about $2.2^{*} 10^{12}$ respectively. Due to the great number of design variables and the non-linear objective function it is hardly possible to find the global minimum. That is why genetic algorithms have been employed for this pure deterministic optimization with an in-house code which is based on SNMmodels.

According to the results of the optimization one blisk prototype has been manufactured with two different blade geometries, another one with actually identical blades. In consequence, two different blade frequencies are theoretically expected in case of the intentionally mistuned blisk, which are exemplarily shown for the first flap mode as relative frequency deviation in Fig. 6a (blue curve) whereas identical blade frequencies should appear for the tuned blisk (Fig. 6b). However, in reality deviations compared with the design intentions are unavoidable (Fig. 6, red curves), e.g. caused by wear of the milling tool or allowable tolerances in general. For details regarding the identification of real mistuning please refer to section 3.1.
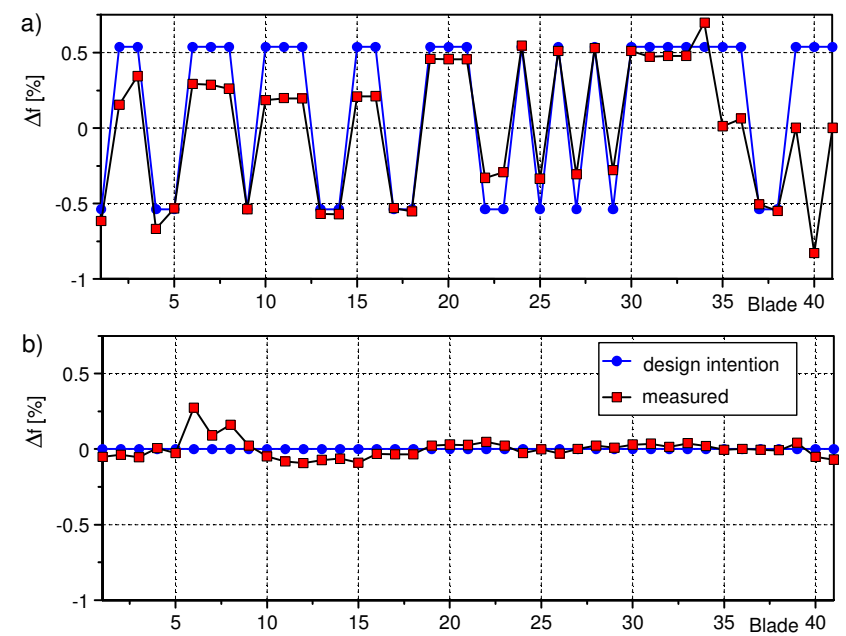

Figure 6. Frequency Mistuning of Fundamental Flap Mode, a) Intentionally Mistuned and b) Tuned [18]

\section{EXPERIMENTAL ANALYSES \\ 3.1 Identification of Frequency Mistuning}

Real frequency mistuning patterns are experimentally determined for both prototypes applying blade by blade tests according to a patented approach introduced by Kühhorn and Beirow ${ }^{[14]}$. In detail miniature hammer excitation is employed for each blade of the blisk step by step. Simultaneously the vibration velocity response of the same blade which is currently excited is recorded non-intrusively by means of laser Doppler vibrometry (LDV). The hammer itself is fixed in a holding device and released by means of electromagnetic control, which enables impacts at the exactly same position and with identical intensity as well (Fig. 7). The blisks are put on foam rubber while testing in order to accomplish approximately free support conditions. Additional mass detuning is employed for the purpose of isolating blade dominated frequencies in the frequency response functions (FRF) by putting individual masses on every blade except the one which is currently excited. In this manner, the slightly disturbed cyclic symmetry due to mistuning is completely destroyed and modal decoupling of blade dominated frequencies is effected by choosing both the quantity and the positioning of the additional mass detuning in a skilful manner depending on the blade mode to be excited. Further efforts are carried on with regard to find an ideal position of excitation which usually represents a compromise between the frequency range to be excited, the mode of interest and the flexibility of the blade. Finally, blade dominated frequencies have to be read out of the FRFs yielding frequency mistuning distributions. Later on these mode-individual mistuning patterns are used as input information for updating structural models.

Frequency mistuning patterns derived in the aforementioned way are displayed in Figs. 8 and 9 for the blade families 1, 2, 3, 5 and 7 for both the blisk with and without intentional mistuning. In general, the mistuning distributions are differing from mode to mode. Starting with the intentionally mistuned blisk and the first blade mode on which the optimization had been focused on, indeed deviations are occurring but the intended mistuning pattern is still largely visible (Fig. 6a). The greatest mismatch can be found at blade 40 and its direct neighbors. The distributions of modes 2 and 3 are quite similar (Fig. 8). Although the pattern of mode 1 is still clearly recognizable at mode 5 increasing differences are appearing. In particular, a drop of the frequency level from blade 34 to 35 is conspicuous, which becomes extreme for mode $7(\sim 3 \%)$, where the overall shape of the mode 1 distribution cannot be recognized any more. In case of the blisk without intentional mistuning similarities regarding the same modes are hardly visible. However, as expected the mistuning strength takes significantly lower values as compared with the intentionally mistuned blisk.

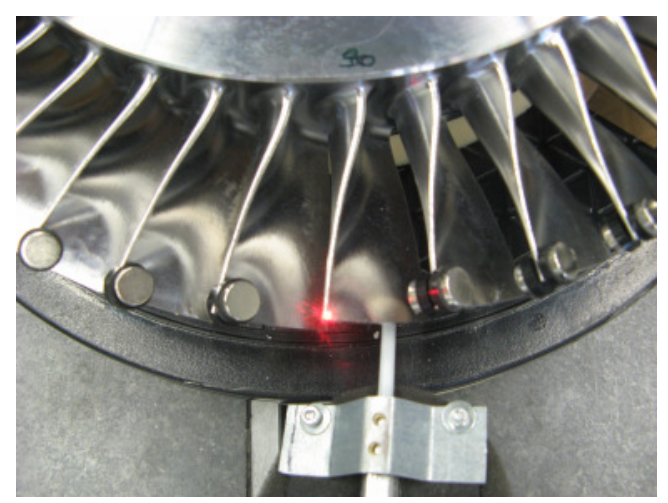

Figure 7. Frequency Mistuning Test with Response Measurement at LE Tip 


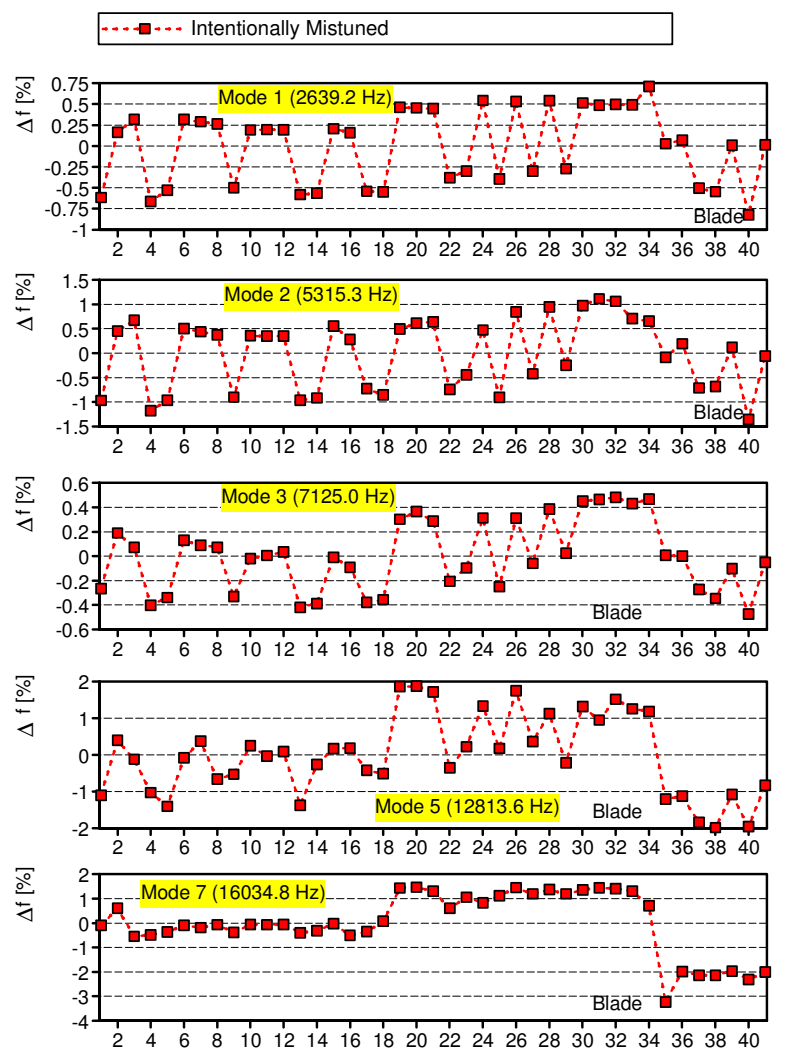

Figure 8. Real Mistuning of Different Blade Modes (Intentionally Mistuned Blisk)

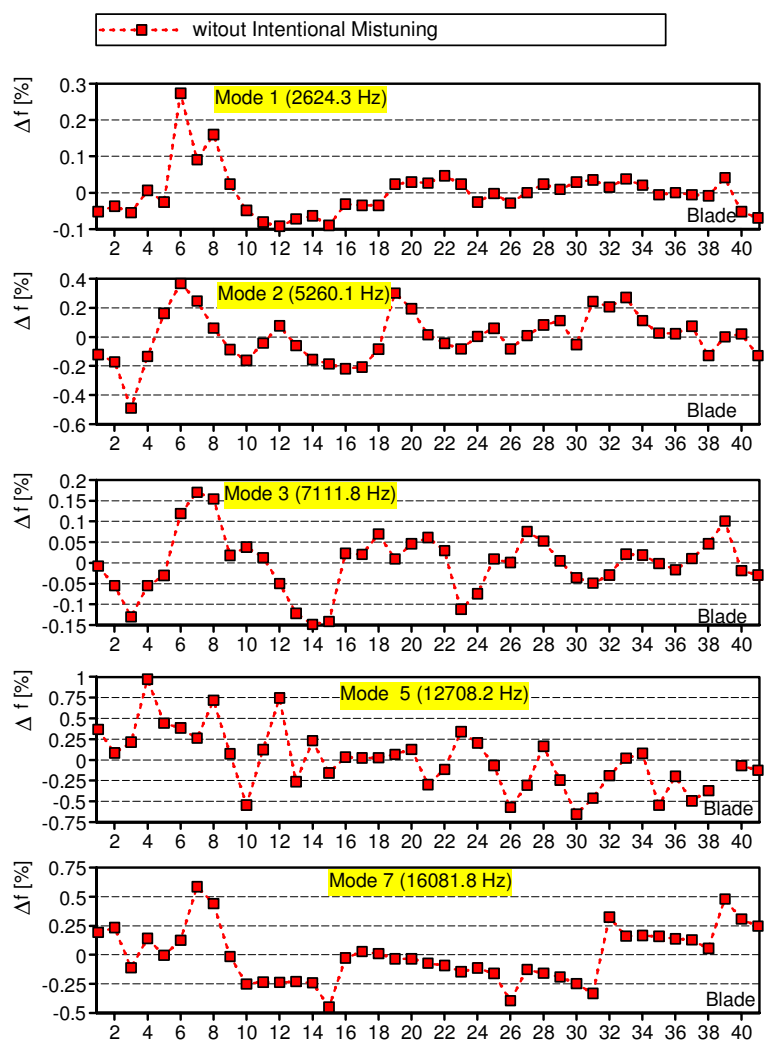

Figure 9. Real Mistuning of Different Blade Modes (Blisk without Intentional Mistuning)

\subsection{Model Updating}

The experimental mistuning data is processed for the purpose of updating structural models. SNM-models have been chosen since they are featuring an extreme low number of DOF and allow for a very simple consideration of blade mistuning. However, SNM-models need to be updated individually for each blade mode $k$. Adjusting the Young's modulus $E_{k, i}$ of every blade $\mathrm{i}$ according to the measured distributions of relative blade dominated frequency deviations is a well established technique in this regard [15]. According to the solution of Kirchhoff-plate's differential equation namely

$$
f_{k, i} \sim \sqrt{E_{k, i}}
$$

it is assumed that the connectivity between deviations of blade dominated frequencies and blade Young's modules of blade i and BF $\mathrm{k}$ can be approximated by

$$
\Delta \mathrm{f}_{\mathrm{k}, \mathrm{i}} \approx \frac{1}{2} \Delta \mathrm{E}_{\mathrm{k}, \mathrm{i}}
$$

If required an additional correction of possible mean frequency shifts can be provided by adjusting the global structural density.

\subsection{Experimental Modal Analyses}

The accomplishment of experimental modal analyses is focused on both the determination of modal data for validating the SNM-models by means of mode shape involutes and to provide modal damping ratios for later forced response analyses in dependence on state variables such as the air density. Hence, the experiments are carried inside a pressure vessel starting at technical vacuum (1 mbar) up to room conditions (1013 mbar). Deviating from the mistuning identification procedure described before piezoelectric excitation of the blisks (Fig. 1) at the disk and LDV response measurement at the blades is applied to derive FRF without any additional mass detuning. Subsequently, MDOF fitting methods have been employed to extract modal ratios.

Examples of experimentally determined FRF are given in Fig. 10 for the BF 3 region of both blisk prototypes. Although the dominance of particular MCSM-peaks is kept, peak frequencies and peak characteristics are clearly differing. In particular the range between MCSM 1 and 2 is significantly varying due to an increasing number of localized mode shapes appearing for the intentionally mistuned blisk (Fig. 10a). The peak labeled with 'lok' is dedicated to the mode shape involute shown in Fig. 15b.

The acoustic impedance $Z_{F}$ (Eq. 3) has been chosen regarding the assessment of the dependency of modal damping ratios and natural frequencies on ambient conditions since it allows for considering the effect of temperature apart from ambient pressure. In this regard the impact of ambient air generally yields linearly increasing modal damping ratios with increasing acoustic impedance (Fig. 11a). The greatest damping ratios and gradients as well are surprisingly found for BF 3, where the modal damping at 1013 mbar 
$\left(Z_{\mathrm{F}}=413.48 \mathrm{~kg} / \mathrm{s} / \mathrm{m}^{2}\right.$ at $\left.\vartheta \approx 20^{\circ} \mathrm{C}\right)$ is 10 times greater than the value at technical vacuum (1 mbar). Comparably low magnifications ranging between 2 and 5 are found for BF 1 and 2. This can be explained by an increasing contribution of aero-acoustic interaction between adjacent blades due to their lower natural frequencies ${ }^{[16]}$.

In addition very moderately decreasing natural frequencies are noticeable with increasing pressure or acoustic impedance, respectively due to the contribution of co-vibrating air masses as exemplarily shown for MCSM 0 in Fig. 11b. As found before for the modal damping ratios the greatest change occurs for BF 3 .

$$
\mathrm{Z}_{\mathrm{F}}=\frac{\mathrm{p}}{\mathrm{R}_{\mathrm{S}} \cdot \mathrm{T}} \cdot \sqrt{\kappa \cdot \mathrm{R}_{\mathrm{S}} \cdot \mathrm{T}}
$$
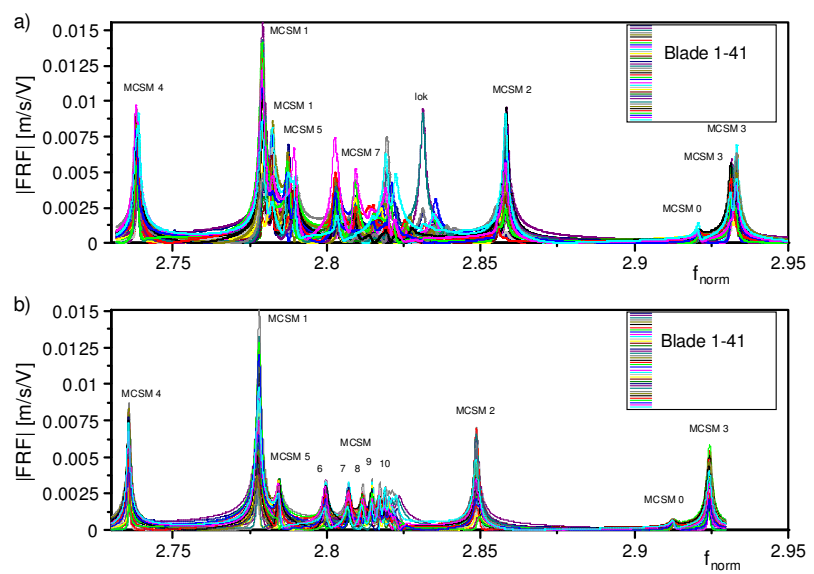

Figure 10. Experimentally Determined FRF BF 3 at 1013 mbar, a) Intentionally Mistuned and b) Tuned
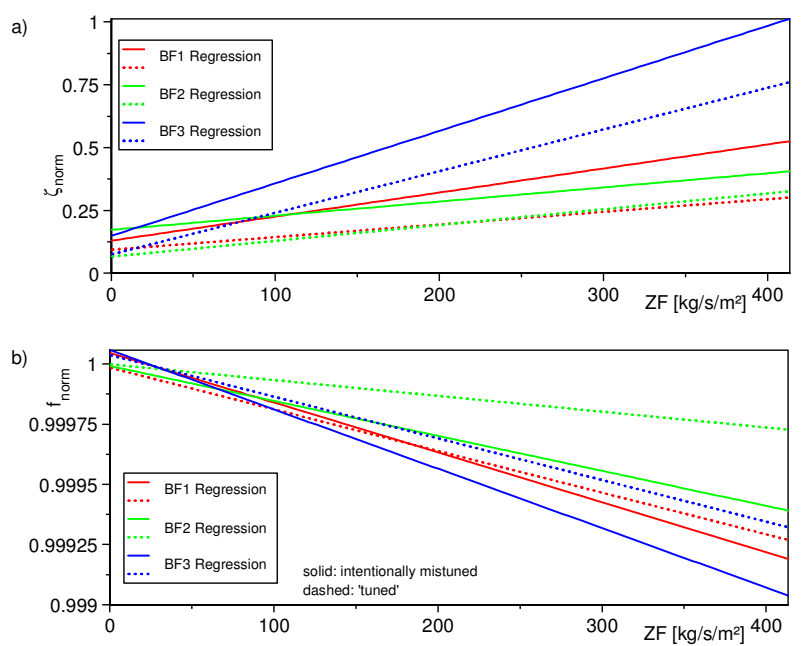

Figure 11. a) Normalized Damping Ratios (Medians) and b) Normalized Natural Frequencies (MCSM 0)

\subsection{Model Validation}

SNM-models have been updated for BF 1, 2 and 3 for both the intentionally mistuned and the actually tuned blisk. The validation of SNM-models is accomplished by comparing computed mode shape involutes with those derived from experimental modal analyses. All in all Figures 12-15 are showing a largely satisfying match for a selection of modes, in particular those representing BF 1 (Figs. 12, 13). This applies to both, modes which are similar to modes expected for the tuned reference (MCSM) and strongly localized mode shapes as seen in Figs. 12b and 13b.

Some characteristics found in the curves are worth to be considered in a deeper discussion. The fluctuations appearing between blades 23 and 30 (Fig. 12c, BF 1/ MCSM 1) are most likely directly correlated with the underlying intentional mistuning distribution (Fig. 6a), which features alternating blade dominated frequencies in the same region. In this regard it has to be mentioned that the frequency splitting caused by mistuning takes the greatest value within BF 1 . A likewise bumpy characteristic of a mode shape involute is also found for BF 3 / MCSM 2 (Fig. 15c) which again features a great frequency splitting of dedicated natural frequencies.
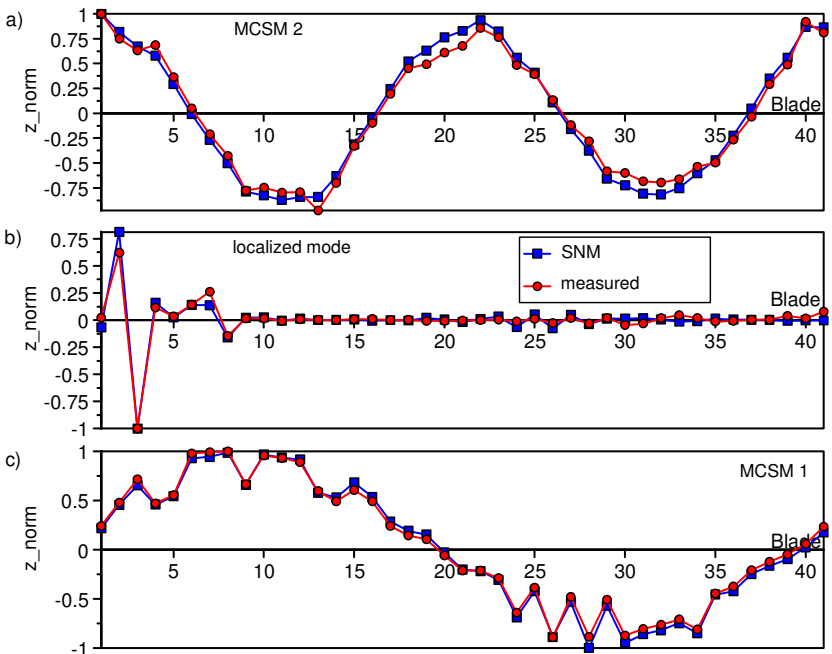

Figure 12. Measured and Calculated Mode Shape Involutes at LE Tip (BF 1, Intentionally Mistuned Blisk)

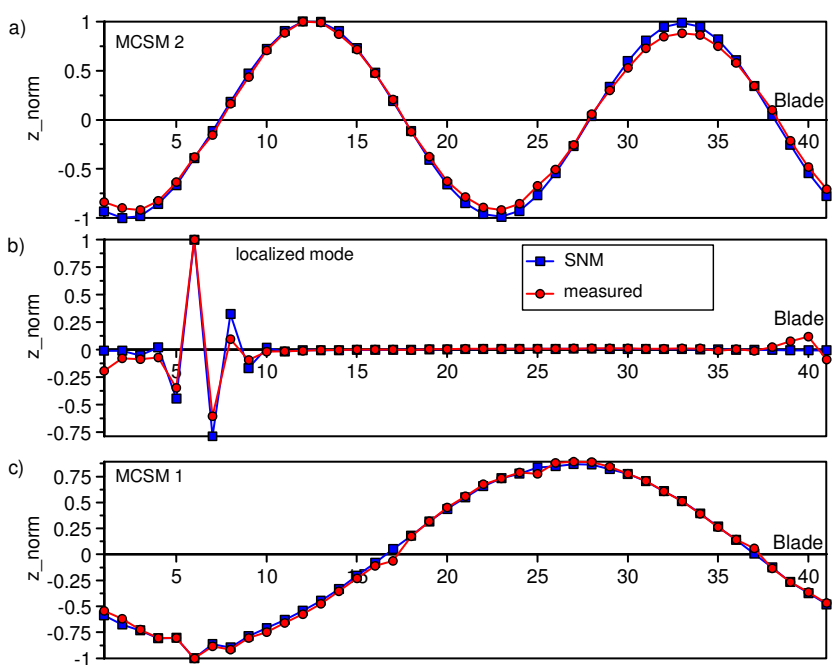

Figure 13. Measured and Calculated Mode Shape Involutes at LE Tip (BF 1, Tuned Blisk) 
Vibration Analysis of an Axial Turbine Blisk with Optimized Mistuning Pattern - 6
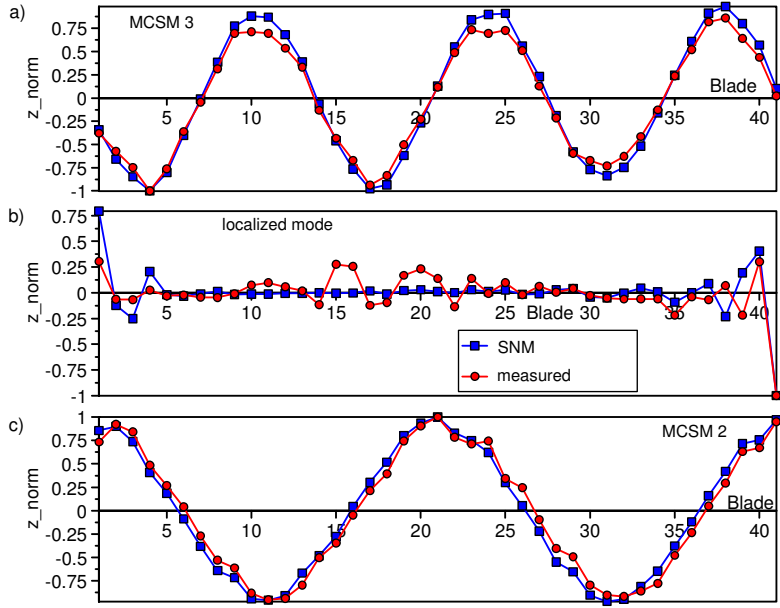

Figure 14. Measured and Calculated Mode Shape Involutes at LE Tip (BF 2, Intentionally Mistuned Blisk)
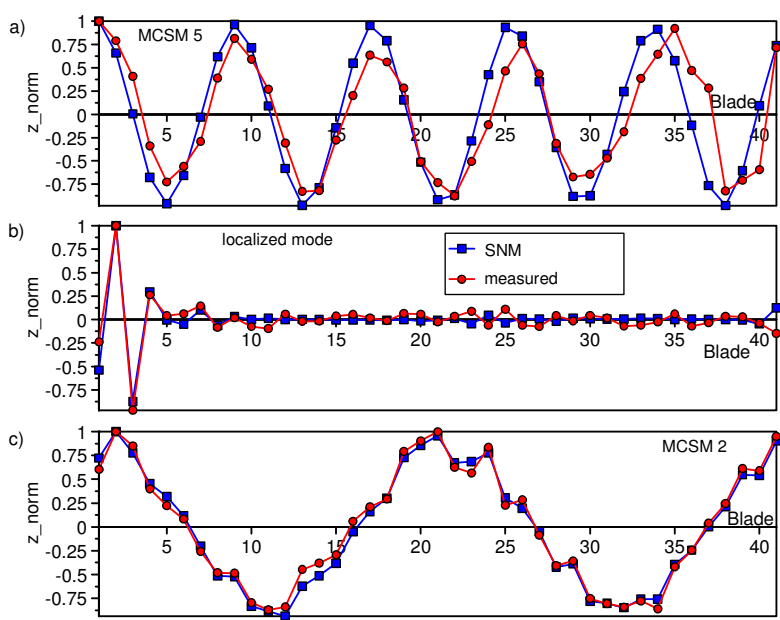

Figure 15. Measured and Calculated Mode Shape Involutes at LE Tip (BF 3, Intentionally Mistuned Blisk)

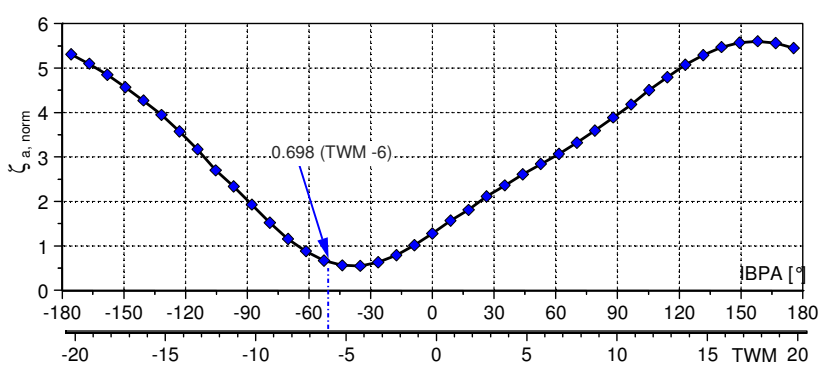

Figure 16. Aerodynamic Damping (Normalized, BF 1, 90\% Speed)

\section{FORCED RESPONSE ANALYSES AND ROBUSTNESS}

Forced response analyses are focused on an EO 6 excitation of the fundamental mode at $90 \%$ speed as indicated in the Campbell diagram (Fig. 5). The aeroelastic coupling between blades is considered by employing AICs, which have been derived from a CFD-simulation regarding the motion of one reference blade in an originally stationary flow field. Following the AICs are incorporated into the SNM, which is only valid for one BF and the operational conditions presumed during the computation of AICs. Small deflections and linear conditions are required within the AIC-approach, in which the intensity of aeroelastic coupling depends on the blade distance. By formulating an eigenvalue problem based on SNM including AICs, aerodynamic damping ratios in dependence on the inter blade phase angle (IBPA) or the travelling wave mode (TWM) respectively can be calculated (Fig. 16). The run of the damping curve is promising with respect to take advantage of mistuning for increasing the resulting aerodynamic damping since it meets the target that both the damping dedicated to the TWM to be excited in case of a tuned blisk (TWM -6) is weakly damped compared to most others and a large difference between maximum and minimum damping becomes apparent. The ratio takes a value of more than 10 in the case under consideration:

$$
\max \zeta_{\mathrm{a}} / \min \zeta_{\mathrm{a}}=10.17
$$

For the forced response analysis four cases are distinguished:

- Ideally tuned blisk

- 'Tuned' blisk as manufactured

- Intentionally mistuned blisk according to optimization

- Intentionally mistuned blisk as manufactured

Results regarding the worst ODS of each case are revealed in Fig. 17. The value $\gamma$ denotes the maximum magnification of the forced response compared to the tuned reference. As can be seen Fig. 17a the ODS dedicated to the maximum tuned response takes a pure sine shape. The sine shape is appearing slightly disturbed for the really manufactured 'tuned' blisk (Fig. 17b) and a moderate increase of maximum magnification is indicated $\left(\gamma_{\max }=1.10\right)$. For the intentional mistuning pattern obtained from optimization the maximum forced response will be tremendously decreased down to a level of $29.4 \%\left(\gamma_{\max }=0.294\right.$, Fig. $\left.17 \mathrm{c}\right)$ compared to the tuned counterpart (100\%). This is a consequence of an increase of the resulting aerodynamic damping ratio by a factor greater than 4 which in turn is explainable with the superposition of the forced response by several TWM instead of only the $6^{\text {th }}$ one as it would occur in case of a tuned blisk (Fig. 18). Since the TWM -6 is dedicated to an aerodynamic damping ratio close to the minimum (Fig. 16), nearly all other TWM participating to the response are contributing additional damping. The resulting aerodynamic damping [18] can be computed by

$$
\zeta_{\text {a,res }}=\frac{\sum_{k=-T W M_{\max }}^{T_{\text {max }} \zeta_{\mathrm{ax}}} \cdot \mathrm{Amp}_{\mathrm{TWM}, \mathrm{k}}}{\sum_{\mathrm{T}=-\mathrm{TWM} M_{\max }}^{\mathrm{TWM}} \mathrm{Amp}_{\mathrm{TWM}, \mathrm{k}}},
$$

which takes a more than 4 times greater value for the optimized mistuning pattern compared to the tuned reference (Table 1). In case of the intentionally mistuned blisk as manufactured even more damping has been activated due to the TWM contribution. Nevertheless, a part of the response reduction gets lost $\left(\gamma_{\max }=0.376\right)$ since the response magnifying effect of unavoidable random mistuning is working against the effect of enhanced damping. 
a)

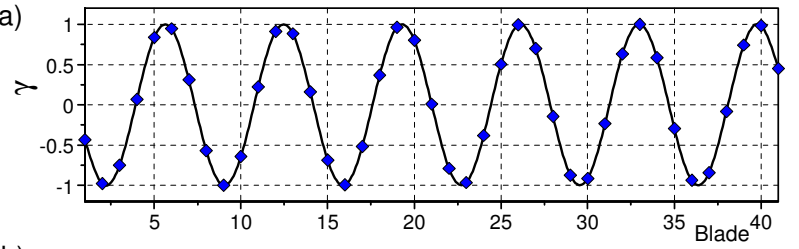

b)

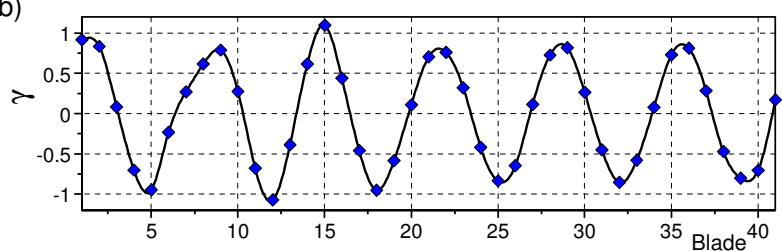

c)

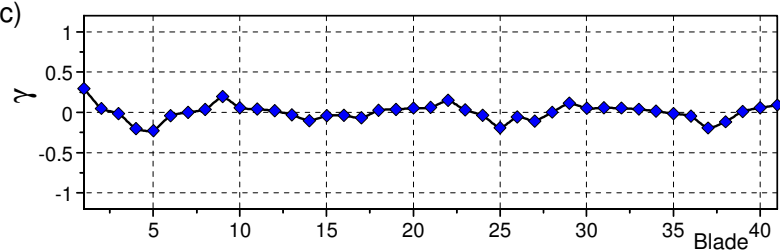

d)

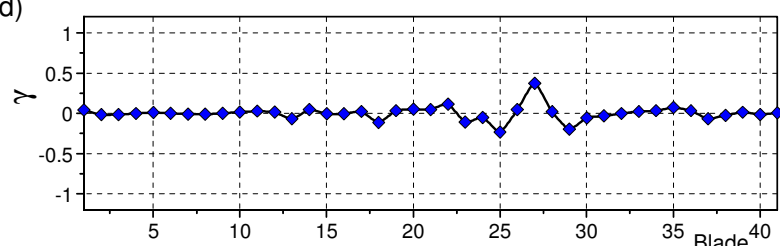

Figure 17. Maximum ODS (EO6, BF 1, 90\% Speed, Aerodynamic Damping as Given in Fig. 16). a) Tuned,

b) 'Tuned' as Measured, c) Optimum Intentional

Mistuning and d) Intentional Mistuning as Measured

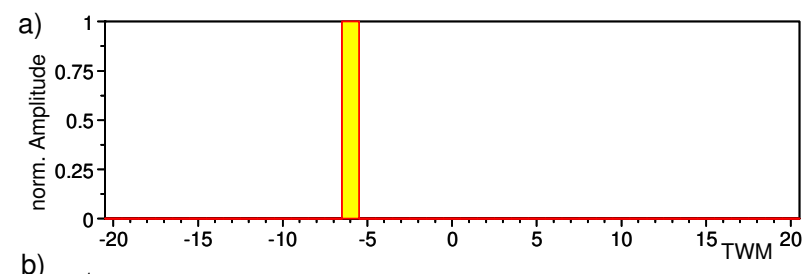

b)
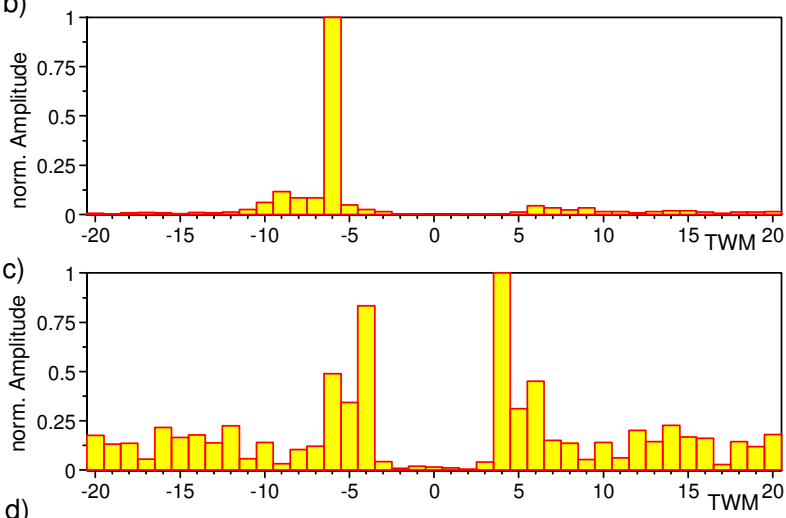

d)

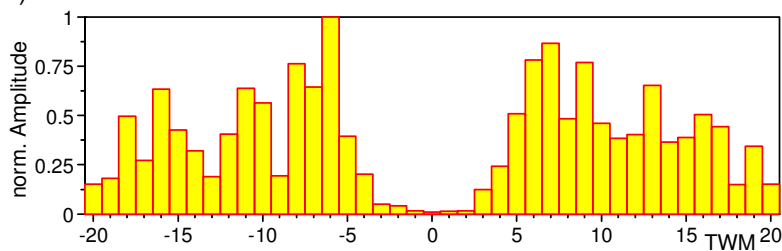

Figure 18. TWM-Development of Maximum ODS Given in Fig. 17)

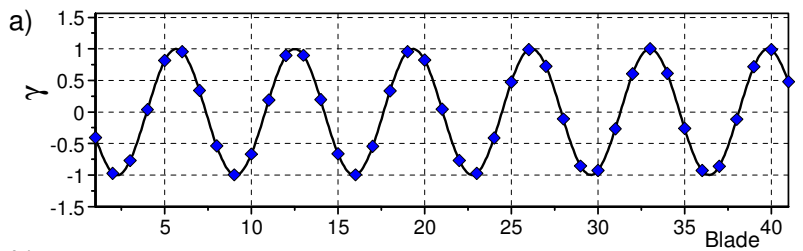

b)
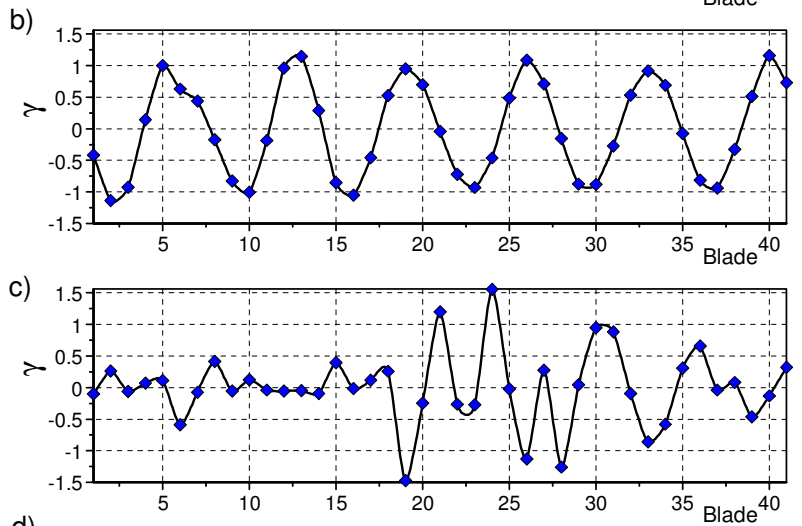

d)

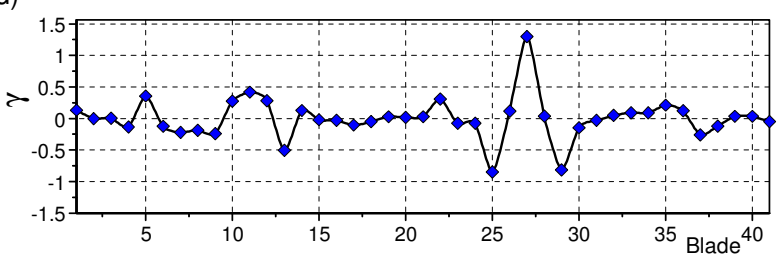

Figure 19. Maximum ODS (EO6, BF 1, 90\% Speed, Constant Aerodynamic Damping Assumed: $\left.\zeta_{\mathrm{a}, \mathrm{norm}}=0.698\right)$. a) Tuned, b) 'Tuned' as Measured, c) Optimum Intentional Mistuning and d) Intentional Mistuning as Measured

Table 1. Increase of damping ratios and response magnification

\begin{tabular}{lllll} 
Blisk & $\gamma$ & $\zeta_{\mathrm{a} \text {,res }}$ & $\begin{array}{c}\zeta_{\mathrm{a}, \text { res }} \\
\text { / }\end{array}$ & $\gamma\left(\zeta_{\mathrm{a}, \text { res,tuned }}=0.698\right)$ \\
\hline Tuned (ref.) & 1.000 & 0.698 & 1 & 1 \\
'Tuned' (real) & 1.100 & 1.520 & 2.18 & 1.157 \\
IM (opt.) & 0.294 & 2.867 & 4.11 & 1.554 \\
IM (real) & 0.376 & 3.230 & 4.63 & 1.298
\end{tabular}

Additional forced response analyses have been carried out for purposes of comparison in which again EO 6 excitations are considered but the aerodynamic damping is kept constant corresponding to the tuned blisk $\left(\zeta_{a, n o r m}=\right.$ 0.698). The results shown in Fig. 19 and Table 1 (last column) as well are indicating that without reducing the resulting aerodynamic damping no reduction of the maximum forced response beyond that of the tuned reference is computed but a general increase. Indeed the difference for the really 'tuned' blisk is small whereas a strong magnification of the response is found for the blisks with intentional mistuning (IM). For the optimized design the magnification takes a value of $55.4 \%$ and completely different ODS are computed (Figs. 17c and 19c). In contrast, more similar ODS are calculated for the IM as manufactured both featuring a localization at blade 27 . The maximum magnification of the response does not exceed 
$30 \%$.

Again considering IBPA-dependent damping the robustness of the gain achieved by intentional mistuning is analysed. For this purpose Monte Carlo simulations have been carried out allowing a particular amount of additional Young's modulus variations of each blade accomplished by the use of uniformly distributed random numbers. For a first study a one percent random variation is chosen which conforms to a frequency variation between $+/-0.5 \%$ (Eq. 2). Figure 20 indicates the maximum displacement magnification (EO 6 at $90 \%$ speed) considering 5000 samples. The greatest magnification is found at blade 7 with $\gamma_{\max }=0.480$, which indeed exceeds the maximum of the optimized intentional mistuning design $\left(\gamma_{\max }=0.294\right)$, however clearly remains below the tuned reference $\left(\gamma_{\max }=1\right)$. The $95 \%$ percentile takes a maximum value of 0.328 at blade 25 . Even if an additional random mistuning of $2 \%$ or even $4 \%$ Young's modulus variation is allowed in a second and third analysis with again 5000 samples each, the $95 \%$ percentiles do not exceed $\gamma_{\max }=0.358$ at the same blade (Fig. 21). Hence, the optimized intentional mistuning pattern proves to be robust towards unavoidable additional random mistuning.

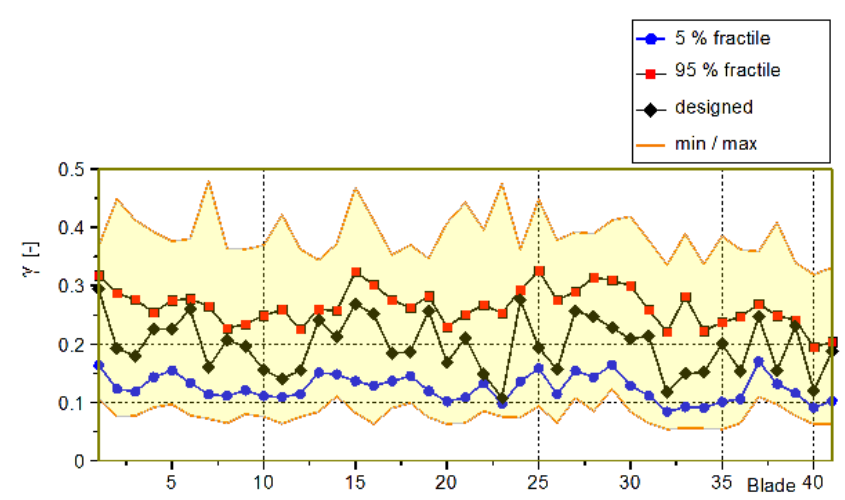

Figure 20. Maximum Displacement Magnification

(EO 6, BF 1, $90 \%$ Speed, Designed Intentional Mistuning Superimposed by $1 \%$ Random Mistuning)

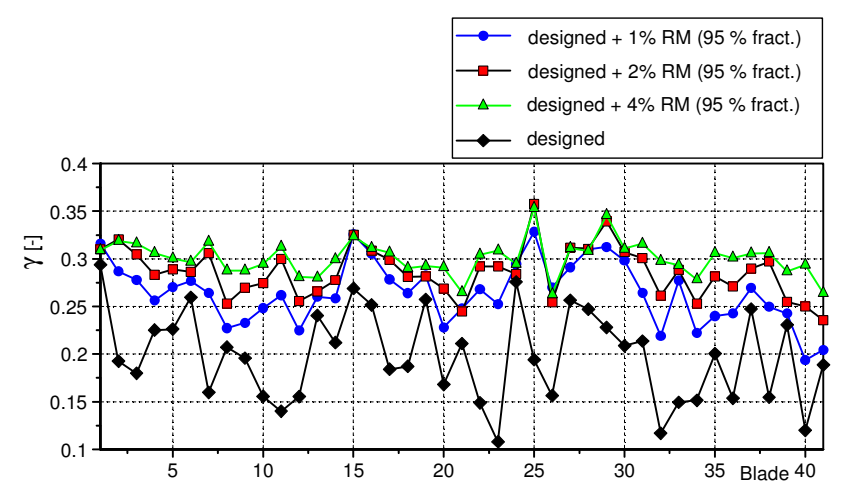

Figure 21. 95\% Percentiles of Maximum

Displacement Magnification (EO 6, BF 1, 90 \% Speed,

Designed Intentional Mistuning Superimposed by

Random Mistuning)

\section{CONCLUSIONS}

Genetic algorithms have been employed to identify an optimal intentional mistuning pattern for an axial turbine blisk yielding a minimized forced response in consequence of LEO. The tremendous response minimization (EO 6, 90\% speed, BF 1) down to about $30 \%$ compared to the tuned reference is mainly achieved by taking advantage from enhancing the aeroelastic interaction in terms of reducing the resulting aerodynamic damping. Both large differences between the IBPA-dependent aerodynamic damping ratios and a small damping ratio of the regularly excited TWM are basic requirements for the success of the approach.

In order to keep the effort for a technical implementation of the mistuning pattern within limits, only two different blade geometries have been admitted for accomplishing the mistuning in this integer optimization problem. With the objective to prove both suitability and robustness of the approach, two blisk prototypes have been manufactured: A first one with the optimized intentional mistuning pattern, a second one without any intentional mistuning. By means of blade by blade measurements the real mistuning could be identified in terms of blade dominated frequencies, which has been subsequently used to update SNM models. These models have been validated by comparing numerical and experimental results of mode shape involutes. Within forced response analyses it could be shown that the effect of unavoidable additional mistuning hardly reduces the gain achieved by the impact of intentional mistuning. In addition, the robustness of the mistuning pattern could be proved by means of Monte Carlo simulations allowing for superimposing additional random mistuning even greater than the intentional mistuning itself.

It could be shown within further experimental investigations carried out for the blisks at rest inside a pressure vessel that the modal damping ratios are linearly increasing with increasing pressure of the ambient air. Modal damping ratios found at 1013 mbar take values which are up to 10 times greater than those determined at technical vacuum. Contrary a slight decrease of natural frequencies has been proved with increasing pressure due to the impact of covibration air mass.

\section{ACKNOWLEDGMENTS}

The research was conducted as a part of the joint research program "SEABLISK". The work was supported by the Bundesministerium für Wirtschaft und Energie (BMWi) under FKZ 03SX335B. The authors gratefully acknowledge MAN Diesel \& Turbo SE for their support and permission to publish this paper. The responsibility for the content lies solely with its authors.

\section{NOMENCLATURE}

AIC Aerodynamic Influence Coefficient

BF Blade Mode Family

CSM Cyclic Symmetry Mode

E Young's Modulus

EO Engine Order 


$\begin{array}{ll}f & \text { Frequency } \\ \text { FRF } & \text { Frequency Response Function } \\ \mathrm{i} & \text { Blade Index } \\ \text { IM } & \text { Intentional Mistuning } \\ \mathrm{k} & \text { BF index, TWM index } \\ \text { LDV } & \text { Laser Doppler Vibrometry } \\ \mathrm{LE} & \text { Leading Edge } \\ \mathrm{LEO} & \text { Low Engine Order Excitation } \\ \mathrm{M} & \text { (Blade) Mode } \\ \mathrm{MCSM} & \text { Modified Cyclic Symmetry Mode } \\ \mathrm{ODS} & \text { Operational Deflection Shape } \\ \mathrm{p} & \text { Pressure [mbar] } \\ \mathrm{R}_{\mathrm{S}} & \text { Specific Gas Constant } \\ \mathrm{RM} & \text { Random Mistuning } \\ \mathrm{SNM} & \text { Subset of Nominal System Modes } \\ \mathrm{T} & \text { Temperature [K] } \\ \text { TWM } & \text { Travelling Wave Mode } \\ Z_{\mathrm{F}} & \text { Acoustic Impedance } \\ \gamma & \text { Max. Blade Displacement Magnification } \\ \zeta & \text { Modal Damping Ratio } \\ \kappa & \text { Ratio of Specific Heats }\end{array}$

\section{REFERENCES}

[1] D. S. Whitehead. Effect of Mistuning on the Vibration of Turbomachine Blades Induced by Wakes, Journal Mechanical Engineering Science, 8, pp. 15-21, 1966.

[2] C. Martel, R. Corral. Asymptotic Description of Maximum Mistuning Amplification of Bladed Disk Forced Response, J Eng Gas Turb Power, 131, 022506-1, pp. 1-10, 2009.

${ }^{[3]}$ F. Figaschewsky, A. Kühhorn. Analysis of Mistuned Blade Vibrations Based on Normally Distributed Blade Individual Natural Frequencies. Proceedings of ASME Turbo Expo 2015, GT2015-43121, 13-19 June 2015, Montréal, Canada, 2015.

${ }^{[4]}$ E. P. Petrov, D J Ewins. Analysis of the Worst Mistuning Patterns in Bladed Disk Assemblies, J Turbomach, 125, pp. 623-631, 2003.

[5] Y. J. Chan. Variability of Blade Vibration in Mistuned Bladed Discs, Ph.D. Dissertation, Imperial College, London, 2009.

[6] R. Bladh, C. Pierre, M. P. Castanier, M. J. Kruse. Dynamic Response Prediction for a Mistuned Industrial Turbomachinery Rotor Using Reduced-Order Modeling. $J$ Eng Gas Turb Power, 124, pp. 311-324, 2002.

[7] J. Judge, C. Pierre. Experimental Investigations of Mode Localization and Forced Response Amplitude Magnification for a Mistuned Bladed Disk. J Eng Gas Turb Power, 123, pp. 940-950, 2001.

${ }^{[8]}$ E. P. Petrov. Reduction of Forced Response Levels for Bladed Discs by Mistuning: Overview of the Phenomenon. Proceedings of the ASME Turbo Expo 2010: Paper GT2010-23299, Glasgow, June 14-18, 2010.

[9] H. Schoenenborn, M. Junge, U. Retze. Contribution to Free and Forced Vibration Analysis of an Intentionally Mistuned Blisk. Proceedings of the ASME Turbo Expo 2012: Paper GT2012-68683, Copenhagen, Denmark, June 11-15, 2012.

${ }^{[10]}$ B. Beirow, A. Kühhorn, T. Giersch, J. Nipkau.
Optimization-Aided Forced Response Analysis of a Mistuned Compressor Blisk. J Eng Gas Turb Power, 137(1): 012504/1-10, 2015.

[11] M. T. Yang, J. H. Griffin. A Reduced-Order Model of Mistuning Using a Subset of Nominal System Modes. J Eng Gas Turb Power, 123: 893-900, 2001.

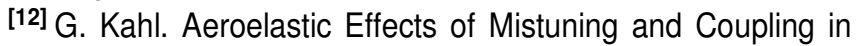
Turbomachinery Bladings". Thèse $\mathrm{N}^{\circ} 2629$, École Polytechnique Fédérale de Lausanne, 2002.

${ }^{[13]}$ F. Figaschewsky, T. Giersch, A. Kühhorn. Probabilistic Analysis of Low Engine Order Excitation Due to Geometric Perturbations of Upstream Nozzle Guide Vanes. Proceedings of 22nd International Conference on Air Breathing Engines 2015, ISABE-2015-20165, 25-30 October 2015, Phoenix, USA, 2015.

${ }^{[14]}$ A. Kühhorn, B. Beirow. Method for Determining Blade Mistuning on Integrally Manufactured Rotor Wheels, Patent US 2010/0286934 A1, 2010.

[15] P. Hönisch, U. Strehlau, A. Kühhorn. Modelling of Industrial Blade Integrated Disks (Blisks) with Regard to Mistuning. Proceedings of ISMA 2012, Leuven, Belgium, 2012.

${ }^{[16]}$ B. Beirow, T. Maywald, F. Figaschewsky, A. Kühhorn, C.R., Heinrich, T. Giersch. Simplified Determination of Aerodynamic Damping for Bladed Rotors. Part 1: Experimental Validation at Rest. Proceedings of ASME Turbo Expo 2016, GT2016-56535, Seoul, South Korea, June 13-17, 2016.

[17] J. Nipkau. Analysis of mistuned blisk vibrations using a surrogate lumped mass model with aerodynamic influences. PhD - Thesis at Brandenburg University of Technology Cottbus, 2010.

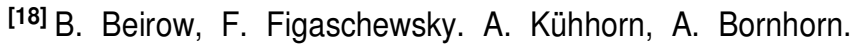
Modal Analyses of an Axial Turbine Blisk with Intentional Mistuning. ASME Turbo Expo, June 26-30, GT201763193, Charlotte, 2017. 